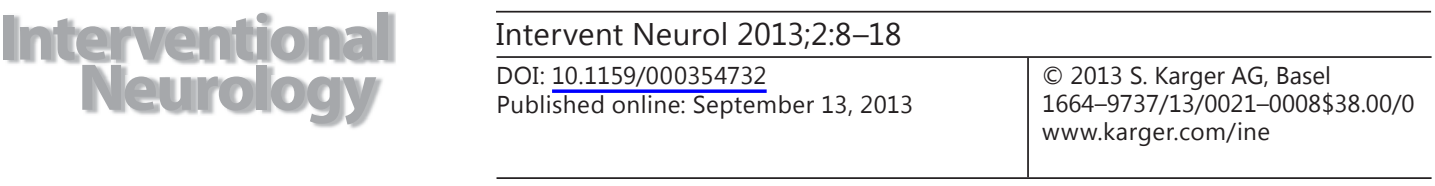

\title{
Usefulness of Transcranial Doppler Ultrasound in Evaluating Cervical-Cranial Collateral Circulations
}

\author{
Jingxia Guan Shaofeng Zhang Qin Zhou Chengyan Li Zuneng Lu \\ Department of Neurology, Renmin Hospital of Wuhan University, Wuhan, PR China
}

\section{Key Words}

Transcranial Doppler · Collateral circulations · Carotid stenosis · Intracranial stenosis

\begin{abstract}
Transcranial Doppler (TCD) ultrasound is a noninvasive, safe and cost-effective bedside test for evaluating cerebrovascular circulation in real time. It has been rapidly evolving from a simple noninvasive diagnostic tool to an imaging model with a broad spectrum of clinical applications. TCD can show the spectral flow waveforms, blood flow direction, velocities and intensity in the intracerebral vessels, adding physiologic information to other imaging models. TCD can also detect collateral channels through the anterior communicating artery, posterior communicating arteries, reversed ophthalmic artery, leptomeningeal collaterals, reversed basilar artery and reversed vertebral artery caused by hemodynamically significant carotid or intracranial stenosis. This article gives a brief overview of its use in evaluation of collateral circulation in carotid and intracranial steno-occlusive disease.

(C) 2013 S. Karger AG, Basel
\end{abstract}

\section{Introduction}

The intracranial collateral channels are dormant under normal circulatory conditions. A collateral channel opens when a pressure gradient develops between the two arterial systems that have anastomoses [1]. Anterior and posterior communicating arteries are considered the primary collateral pathways. The ophthalmic artery and blood flow via leptomeningeal vessels are considered the secondary pathways [2]. Collateral flow helps to maintain cerebral perfusion in the setting of arterial occlusion. Several studies have established the importance of collateral flow in predicting stroke outcome by correlating the degree of collateral circulation with infarct volume and functional status [3-5]. Knowledge of the collateral ability is 
important for neurosurgeons, vascular surgeons and interventional radiologists when a procedure in the intracranial or extracranial cerebral arteries is to be attempted.

Conventional angiography provides the most reliable information about the circle of Willis and leptomeningeal collaterals and is, therefore, considered the gold standard for collateral flow assessment [6,7]. However, the applicability of catheter angiography is limited by its invasive nature and associated risks. Indirect assessment of collaterals can be accomplished by transcranial Doppler (TCD) ultrasound. TCD has been recommended by the American Association of Neurology for the evaluation of the collateral pathways in the condition of an internal carotid artery (ICA) occlusion [8]. The great advantage of TCD is its noninvasiveness and its ability for repeated examinations. In chronic cerebrovascular stenoocclusive disease, TCD is nowadays routinely used in many centers for screening of ICA stenosis [9-11]. Furthermore, it can be used for follow-up evaluations of cerebral collaterals after vascular interventions such as thrombolysis, carotid endarterectomy or stenting [12, 13]. TCD can directly detect the following collateral channels.

\section{Collateral Flow via the Circle of Willis}

TCD is used primarily for evaluation of collateral routes at the circle of Willis. The circle of Willis, located at the base of the brain, provides the main route for collateral blood flow in severe occlusive disease of the ICA by anterior communicating artery (ACoA) and posterior communicating artery (PCoA).

\section{Collateral Flow through the ACoA}

Collateral flow through the ACoA cannot be reliably distinguished from the neighboring $A_{1}$ and $A_{2}$ segments of the anterior cerebral artery (ACA) due to the smaller ACoA length and diameter compared to a large sample volume with which ultrasound intercepts this area. TCD can only determine flow findings consistent with anterior cross-filling via the ACoA rather than measure flow velocity in the ACoA itself (fig. 1). In patients with hemodynamically significant ICA disease, TCD may demonstrate the following indirect signs indicating ACoA filling [14, 15]: (1) a decrease in mean flow velocity (MFV) and pulsatility in the ipsilateral middle cerebral artery (MCA) together with normal flow in the contralateral MCA; (2) reversed direction of flow in the ipsilateral $A_{1}$ segment of the ACA; (3) no significant change in MFV of the ipsilateral MCA and $\mathrm{A}_{1}$ ACA by compression test of ipsilateral common carotid artery, but decreased MFV by compression test of contralateral common carotid artery, and (4) increased blood flow in contralateral $\mathrm{A}_{1}$ ACA, presenting as MFV 1.2 times greater than the ipsilateral $\mathrm{A}_{1}$ ACA. Sometimes, a high-velocity jet with bruit can be found at midline depth, highly suggestive of flow interception through ACoA. Compression tests are especially helpful in the evaluation of collateral blood supply originating through ACoA. One must keep in mind the risk of complications of compression, however, especially the possibility of thromboembolic material from an ICA plaque [16]. Referring to these TCD criteria, a highly significant correlation was reported between TCD and cerebral angiography for evaluating the collateral supply through the ACoA. Sensitivity of TCD was 95\% and specificity was $100 \%$ [17].

\section{Collateral Flow through the PCoA}

The PCoA connects the posterior and anterior cerebral arterial systems. PCoA is located at 60-72 mm depths via transtemporal approach (fig. 1). Identification of PCoA signals may represent a formidable challenge as it is difficult to differentiate between PCoA, terminal ICA and compensatory velocity increase in the posterior cerebral artery (PCA). PCoA flow signals can be identified by TCD because the artery can be $0.5-2 \mathrm{~cm}$ long. When the PCoA carries 
Guan et al.: Usefulness of Transcranial Doppler Ultrasound in Evaluating Cervical-Cranial Collateral Circulations

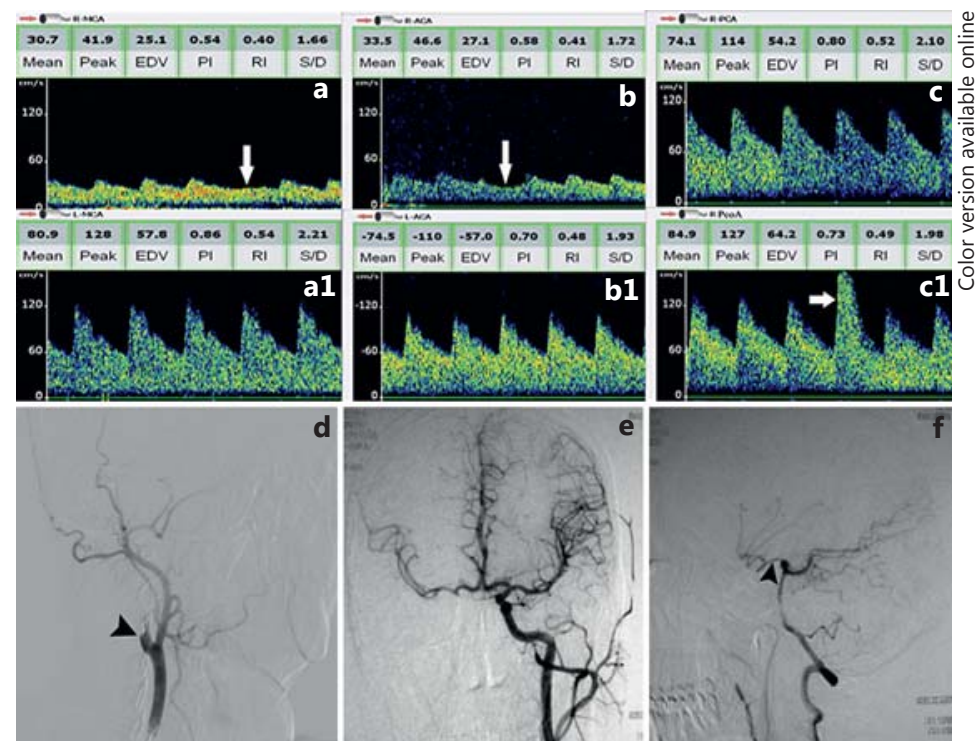

Fig. 1. Collateral flow via the circle of Willis in a patient with right ICA occlusion. The red arrow in the left upper corner of every TCD registration marks the blood flow direction. Arrows pointing to the right indicate flow towards the probe and those pointing to the left indicate flow away from the probe. a Low MFV and lowresistance flow is observed in right MCA spectra with a decreased MFV by compression test of left common carotid artery (white arrow). a1 TCD shows a normal flow velocity and waveforms in left MCA. b Insonating from right temporal window, the direction of flow in the $\mathrm{A}_{1}$ segment of right ACA is reversed (towards probe) with a decreased MFV by compression test of left common carotid artery (white arrow). b1 Increased blood flow in the $A_{1}$ segment of left ACA is observed, combined with spectral changes of both right MCA and ACA, indicating collateral blood flow from the left ICA via the $A_{1}$ segment of the left ACA and ACoA to the $A_{1}$ segment of right ACA and right MCA. c MFV of the right PCA is significantly increased. c1 A high-MFV flow of right PCoA can be detected with a significantly increased flow by compression test of the left common carotid artery (white arrow), suggesting a patent PCoA. d-f Corresponding angiography confirms the TCD recordings. $\mathbf{d}$ The lateral view shows the occlusion of the proximal right ICA (black arrowhead). e The anteroposterior view shows the ACA and MCA perfusion from left ICA via the AcoA. $\mathbf{f}$ Angiography via VA shows PCoA filling (black arrowhead), providing perfusion for anterior circulation.

collateral flow, TCD may demonstrate the following signs [14, 15]: (1) a PCoA flow signal consistently presents at varying depths from 55 to $70 \mathrm{~mm}$ via the transtemporal approach when the sonographer switches from the ICA bifurcation posteriorly to locate the PCA; (2) a basilar artery (BA) blood velocity $>70 \mathrm{~cm} / \mathrm{s}$; (3) a marked increase of BA blood velocity after compression of the nonoccluded carotid artery, and (4) an evident side-to-side asymmetry of the blood velocity of the PCAs with a high blood velocity ipsilateral to the ICA occlusion. Comparing these TCD criteria with the corresponding angiographic findings, the correlation was highly significant. The sensitivity of TCD for evaluating the collateral supply via PCoA was $87 \%$ and its specificity was $95 \%$ [17]. The evaluation of the collateral supply through the PCoA by TCD is more difficult and sometimes more time-consuming than the evaluation of the collateral flow through the ACoA [18].

Studies that have compared TCD with magnetic resonance angiography (MRA) also show a high correlation in the prevalence of collateral flow via the PCoA and/or ACoA $[19,20]$. Patients with TCD signs of established collateral flow via ACoA or PCoA generally show highgrade stenosis ( $>70 \%$ ) both on digital subtraction angiography (DSA) and on MRA [18]. TCD is very sensitive to hemodynamic changes associated with severe $(>70 \%)$ carotid stenosis or 
Guan et al.: Usefulness of Transcranial Doppler Ultrasound in Evaluating Cervical-Cranial Collateral Circulations
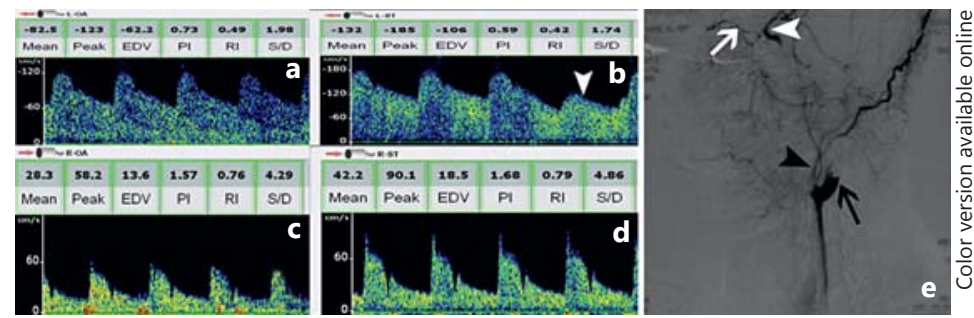

Fig. 2. Reversed OA flow in a patient with proximal ICA occlusion. a Flow direction of left OA is reversed (away from probe) and pulsatility index is significantly decreased (0.73). b Flow direction of left supratrochlear artery is also reversed with decreased pulsatility index, which is consistent with OA spectra. Flow velocity of left supratrochlear artery significantly reduced by compression of ipsilateral superficial temporal artery and mandibular artery (white arrowhead), indicating which is supplied by ECA. c, $\mathbf{d}$ TCD shows a normal flow velocity and waveforms in right $\mathrm{OA}$ and supratrochlear artery, respectively. e Corresponding angiography confirms the TCD recordings. Cerebral angiography shows left proximal ICA occlusion (black arrow). Blood source of OA (white arrow) is from ECA (black arrowhead). OA provides blood supply for distal ICA (white arrowhead), indicating ECA to ICA collateral channels filling.

occlusion. For a 70-99\% proximal ICA stenosis and occlusion, TCD had sensitivity of 79\%, specificity of $86 \%$, positive predictive power value (PPV) of $57 \%$, negative predictive value (NPV) of $95 \%$ and accuracy of $85 \%$. The degree of $50-69 \%$ ICA stenosis does not result in a significant blood flow reduction and intracranial hemodynamics may not be affected. Therefore, the PPV for these lesions are low [21]. In TCD studies of patients with a unilateral ICA occlusion, the proportion of patients with collateral flow via the $A_{1}$ segment $\left(A_{1}\right.$ segment only or $\mathrm{A}_{1}$ segment plus $\mathrm{PCoA}$ ) varied between 46 and $80 \%$, whereas the prevalence of collateral flow via the PCoA (PCoA only or PCoA plus $\mathrm{A}_{1}$ segment) varied between 32 and $76 \%$ [22]. Ringelstein et al. [23] described a hierarchy of collateral pathways to the brain, indicating a key role of the ACoA in compensating for ICA occlusion. The PCoA emerged as a second-ranked, somewhat less effective pathway. More recently, it was remarked that collateral flow through the PCoA alone is a sign of deteriorated cerebral perfusion. On the other hand, it has been reported that only a small or absent PCoA can be considered as a significant risk factor for watershed infarctions [24].

\section{Reversed Ophthalmic Artery Flow}

Flow in the opthalmic artery (OA) can be reliably assessed with TCD. An abnormal OA signal includes low pulsatility flow directed primarily away from the probe via a transorbital window at 40-60 mm depth (fig. 2). If the reversed $O A$ is the only abnormal finding, this indicates possible ICA occlusion or severe stenosis proximal to OA origin. Occasionally, this may be the only sign of an ICA dissection or occlusion. If a reversed OA is found with a delayed systolic flow acceleration in the unilateral MCA, there is a probable proximal ICA occlusion or severe stenosis, and collateralization capacities through the circle of Willis are likely suboptimal. If a reversed $\mathrm{OA}$ is found with at least one other collateral channel, there is a definite proximal ICA occlusion or a high-grade stenosis $[14,15]$.

Reversed OA flow at 50-60 mm depth revealed high specificity (100\%) and good sensitivity $(75 \%)$ for identifying proximal ICA occlusion or critical stenosis $(\geq 95 \%)$ compared with conventional angiography [25]. OA flow reversal on TCD is an important marker for high-grade ICA stenosis or occlusion, which is never reversed ipsilateral to arteries with 
Guan et al: Usefulness of Transcranial Doppler Ultrasound in Eval

$<80 \%$ stenosis with a sensitivity of $55 \%$, specificity of $100 \%$, NPV of $82 \%$ and PPV of $100 \%$ for OA flow reversal as a marker of high-grade carotid lesions [26]. Furthermore, a normal OA direction does not rule out the proximal ICA stenosis. When OA flow was anterograde, low pulsatility index $(<1.2)$ and MFV $(<15 \mathrm{~cm} / \mathrm{s})$ discriminated critical severe ICA stenosis or occlusion with good sensitivity (87.2\%) and specificity (95.2\%) [25]. Without significant ICA disease, the reversed $\mathrm{OA}$ was found in only $5.6 \%$ of patients and may be attributed in large part to a technical error in vessel identification [11].

The supratrochlear artery is a branch of the OA. It can be easily detected with continuouswave Doppler sonography at the medial canthus. When the OA flow is reversed, flow direction of supratrochlear artery is usually reversed with decreased pulsatility index (fig. 2). In addition, MFV of the supratrochlear artery was significantly reduced by compression of the ipsilateral or contralateral superficial temporal artery and the mandibular artery, which are branches of the external carotid artery (ECA), indicating ECA to ICA collateral channels filling [27].

Collateral flow via the reversed OA flow is often regarded as a marker of insufficient cerebral perfusion and possibly impaired intracranial vasomotor reactivity, which is the last resort of collateral blood flow circulation and is recruited only if both communicating arteries of the circle of Willis are unavailable or insufficient in ICA disease; it usually carries a poor prognosis [28]. A reversed OA flow in the setting of an asymptomatic stenosis may place the patient in a higher-risk group for future ischemic events [29]. However, some have suggested that reversed OA flow does have a functional contribution to cerebral circulation [30, 31]. In conclusion, a reversed OA flow is highly specific for identifying proximal ICA occlusion or severe stenosis.

\section{Leptomeningeal Collaterals in MCA Occlusion}

In patients with proximal MCA occlusion, leptomeningeal collaterals are the only pathways connecting distal MCA branches and other major cerebral arteries. In MCA occlusion, the perfusion pressure in distal MCA branches with proximal occlusion becomes low compared to the adjacent ACA or PCA. So the pressure gradients between ACA/PCA and MCA will increase. These increased pressure gradients may facilitate the opening of leptomeningeal collaterals. The luminal diameter of collateral vessels increases to supply a sufficient blood perfusion. These collateral vessels may work as an alternative pathway to the ACA/PCA, and they might decrease the outflow resistance of the ACA or PCA [1]. So this mechanism increases the flow velocity of ipsilateral ACA/PCA compared to the contralateral side. This can be seen as high-velocity and low-resistance flow in the ipsilateral ACA or PCA in patients with MCA occlusion on TCD examination (fig. 3). This characteristic pattern of cerebral flow dynamics on TCD was previously termed flow diversion (FD) [32-36].

The definition of FD in the literature varies. Some studies used the flow velocity of occluded MCA as a reference velocity [32-36]. Other studies used the contralateral homologous artery as a reference to define FD [37-39]. The former had some ambiguity in the definition of FD, because occluded MCA must have a low flow velocity, which might be lower than that of the ipsilateral ACA. It may cause a high incidence of FD, so it may be reasonable to use the contralateral homologous artery as an ideal reference to define FD. It was shown that a $30 \%$ velocity difference between the right and left ACA had good correlation with the leptomeningeal collateral circulation in angiography [40]. FD was noted more frequently in ACA than in PCA. This can be explained by cortical arterial anastomoses among distal segments of major cerebral arteries. The number and size of anastomotic vessels are greater between ACA and MCA than connections between PCA and MCA. ACA is the major source of leptomeningeal collateral circulation in patients with MCA occlusion, even though the PCA supplies a leptomeningeal collateral in a few cases [1]. 
Guan et al.: Usefulness of Transcranial Doppler Ultrasound in Evaluating Cervical-Cranial Collateral Circulations

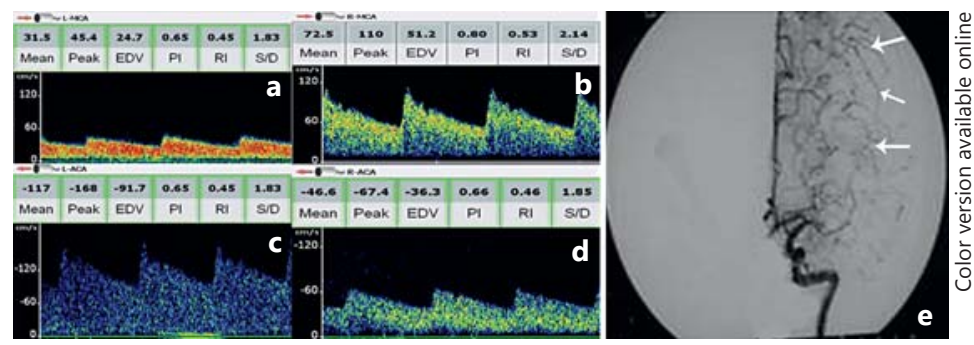

Fig. 3. Flow diversion in the left ACA in patients with left MCA occlusion. a TCD shows a low MFV and low resistance flow in the left MCA. b TCD shows a normal flow velocity and waveforms in right MCA. c, d TCD shows a flow diversion to the left ACA. Flow velocity of the left ACA (MFV: $117 \mathrm{~cm} / \mathrm{s}$ ) is $>30 \%$ higher than that of the right ACA (MFV: $46.6 \mathrm{~cm} / \mathrm{s}$ ). e Angiography shows an occlusion in the left MCA and well-developed leptomeningeal collateral channels (white arrows) from the ACA to the distal MCA branches.

It was reported that the presence of FD showed good correlation with the presence of leptomeningeal collaterals on DSA in patients with MCA stenosis. Sensitivity of FD for leptomeningeal collateral circulation was $81 \%$, specificity $76.7 \%$, PPV $70.8 \%$ and NPV $85.2 \%$ [38]. Many studies show that the presence of leptomeningeal collaterals is correlated with good clinical outcomes in patients with acute ischemic stroke [4, 5]. FD was associated with good collateral flow on computed tomography angiography (CTA) and was an independent predictor of admission infarct core volume and 24-hour infarct volume. The likelihood of a favorable outcome (modified Rankin score 0-2) was higher in those with FD [39]. This also illustrates that FD has effects similar to the leptomeningeal collaterals on clinical outcomes. Acute bedside TCD assessment of FD aids prognostication and therapeutic decision-making in anterior-circulation stroke.

The mechanisms of development of TCD FD and leptomeningeal collateral circulation are similar, as is the biological role they play in patients with MCA occlusion. FD may indicate that collateral channels are active and carrying substantial blood flow to the hypoperfused area distal to the MCA occlusion. FD is an analog of leptomeningeal collateral circulation on DSA or CTA and provides evidence of active leptomeningeal collateral circulation. TCD can provide noninvasive evaluation for leptomeningeal collaterals [41].

\section{Reversed BA Flow}

Acute BA occlusion is a potentially disastrous disease and is often associated with high mortality or poor functional outcome in survivors. Reliable and quick diagnostic techniques are therefore necessary not only for fast confirmation of BA occlusion but also for identification of those patients at a higher risk of poor outcome as candidates for interventional treatment. Flow in the BA can be reliably assessed with TCD. The direction of a normal BA flow is away from the probe via the transoccipital window at a depth of 80-110 mm. TCD can perform more detailed bedside studies of the BA in patients with acute posterior circulation strokes. It can also be used for quick and reliable detection of BA occlusion and flow reversal in its distal portion.

The following criteria are used to identify the reversed BA flow: (1) detectable flow towards the probe in the BA with low-resistance flow at depths of 80-110 mm; (2) absence of the antegrade low-resistance basilar flow signals during suboccipital insonation indicating a proximal BA obstruction; (3) decreased MFV and high-resistance flow in both vertebral 
Fig. 4. Reversed BA flow with proximal basilar occlusion. a, b Low MFV and high-resistance flow in the atlas segment of both VAs detected with continuouswave doppler sonography at the mastoid is consistent with severe distal steno-occlusive disease. c Flow spectra obtained from the right PCoA is away from probe and with transmitted tapping (white arrowhead) of the right common carotid artery in the neck, indicating provide blood supply for posterior circulation. d Reversed flow is observed in BA with transmitted signals during tapping of the common carotid arteries in the neck (white arrowhead), indicating supply by carotid system. e Axial $\mathrm{T}_{2}$-weighted MRI shows brainstem infarction. Flow void of BA disappeared and filled with high signal (black arrowhead), indicating occlusion of the BA. $f$ MRA shows prominent PCoA (black arrows) and proximal occlusion of BA (white arrowhead).

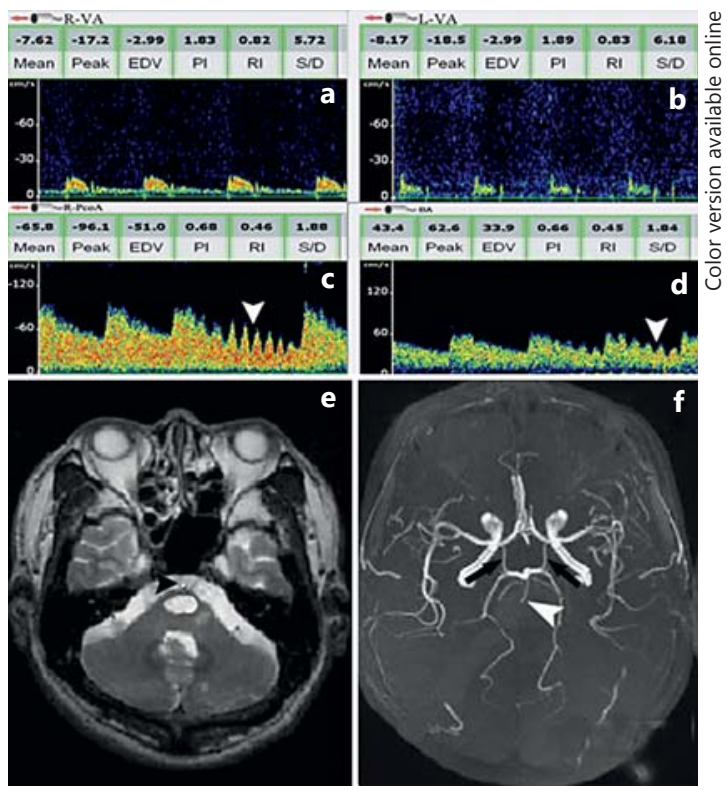

arteries (VAs); (4) anterior circulation flow origin at the top of the BA demonstrated by vertical systolic flow acceleration similar to that in the ICA [14, 15, 42], and (5) the transmitted signals in the PCoA as well as the BA as a response to the common carotid artery tapping (fig. 4). Of note, common carotid artery tapping should be performed with caution, preferably after the extracranial ultrasound examination has confirmed the absence of significant stenosis or hypoechoic plaques in the carotid arteries.

The reversed BA flow indicates collateralization of the blood flow through the PCoA and good prognosis in proximal basilar occlusion [43]. This reversed flow develops because of a pressure gradient. If occlusion develops in the proximal BA, a pressure gradient develops between carotid circulation, and the PCA, superior cerebellar arteries and perforating vessels may recruit PCoA and deliver blood from carotids via the reversed basilar stem to parts of the cerebellum and smaller distal basilar branches. In the setting of bilateral vertebral critical stenosis and occlusion, the proximal BA could have a reversed flow direction supplied by carotid arteries via the PCoA. This collateral flow indicates continuing perfusion of vital brain structures and is associated with lower stroke severity and better outcome after acute proximal BA occlusion. It has been shown that early collateralization of blood flow through both PCoAs and perfusion of the brainstem via a reversed flow in the BA results in a good functional recovery [44]. This collateral can explain the heterogeneity of clinical syndromes associated with BA occlusion and may provide some insights as to how patients respond to intra-arterial rescue or conservative management.

\section{Reversed VA Flow}

The VA can be assessed by TCD with flow direction away from the probe via the transoccipital window at a depth of 50-70 mm. In the most common condition, reversed VA flow can be a collateral pathway in patients with complete subclavian steal resulting from occlusion of the proximal subclavian or innominate artery. In the most common form of a vertebrovertebral collateral circulation, the obstruction is bypassed by retrograde blood flow from the 
Guan et al.: Usefulness of Transcranial Doppler Ultrasound in Evaluating Cervical-Cranial Collateral Circulations

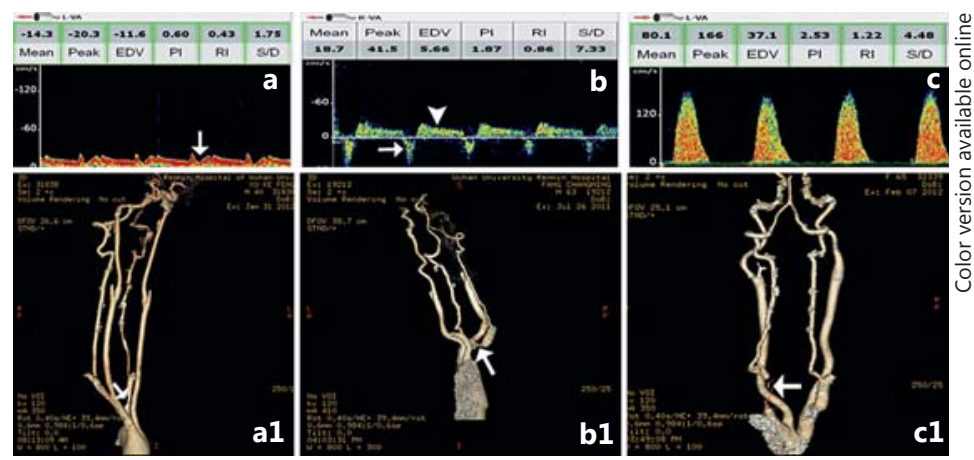

Fig. 5. Reversed VA flow. a TCD spectra of left VA show systolic deceleration (white arrow). a1 Corresponding CTA shows mild stenosis of left subclavian artery (white arrow). b TCD spectra of right VA show reversed flow direction during the systolic period (white arrow) but normal flow direction in the diastolic period (white arrowhead). b1 Corresponding CTA shows moderate stenosis of right subclavian artery (white arrow). c Complete reversed flow direction of left VA during the whole cardiac cycle. c1 Corresponding CTA shows near occlusion of left subclavian artery (white arrow).

ipsilateral VA into the subclavian artery distal to the occlusion, and the reversed flow is fed by the contralateral vertebral artery. If the capacity of the contralateral vertebral artery is also reduced by stenosis or if this artery is occluded, other collateral circulation can arise from external carotid branches (occipital artery) that anastomose with muscular branches of the VA, and anastomoses with the thyrocervical and costocervical trunks may develop $[45,46]$.

The degree of flow reversal in the VA may vary according to the severity and extent of the subclavian arterial stenosis or occlusion [47]. To summarize, TCD spectra are divided into 3 grades depending on the severity of the hemodynamic effects: grade 1 is when there is only systolic deceleration in case of mild-grade proximal subclavian arterial stenosis, grade 2 is when there is a reversed direction of flow during the systolic period but a normal direction in the diastolic period in the case of moderate-grade proximal subclavian arterial stenosis and grade 3 is when there is a permanently reversed blood flow in the VA throughout the whole cardiac cycle in the case of high-grade proximal subclavian arterial stenosis or occlusion (fig. 5).

If flow reversal of VA is incomplete (grade 1 and grade 2), the hyperemia test should be performed to provoke the steal and to augment flow reversal (fig. 6). The cuff should be inflated to over-systolic BP values and flow reduction to the arm should be maintained for about 1-1.5 min (maximum $3 \mathrm{~min}$, if tolerated by the patient) with the patient being asked to clench the fist without intermission. This duration of arterial compression produces ischemia in the arm. The cuff should be quickly released and any augmentation of flow should be monitored by TCD [48]. Once the cuff has been released, the blood flow enters tissues with increased metabolic demand produced by a short period of ischemia. Greater demand for blood flow augments the steal and alternating flow can be visualized for a short period of time in the recipient VA.

\section{Limitations of TCD}

At least three methods of noninvasive imaging have been developed to assess the intracranial hemodynamics. MRA, CTA and TCD are now robust techniques. MRA and CTA are by far more expensive and time-consuming, and require at least some patient cooperation. TCD is cheaper and relatively easy to perform, but is unable to detect the morphological imaging 
Fig. 6. Hyperemia test. a The flow direction of VA changed from systolic deceleration to reversed flow direction during the systolic period but normal flow direction in the diastolic period when overinflated blood pressure cuff released (white arrow). b Reversed flow direction during the systolic period but normal flow direction in the diastolic period changed to complete reversed flow when cuff released (white arrow).

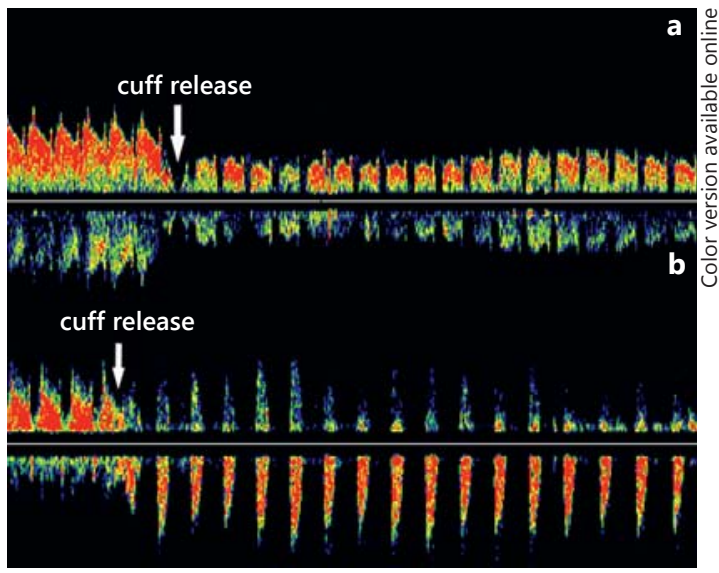

of the brain vessel. However, two major limitations of TCD impede its more widespread use. It is highly operator-dependent and sonographers must have a thorough knowledge of cerebrovascular anatomy and physiology, vascular flow hemodynamics and spectral waveform analysis. TCD is also hampered by the $10-15 \%$ rate of inadequate temporal windows most commonly seen in blacks, Asians and elderly female patients.

Several new techniques in improving and enhancing TCD functions have been developed such as power M-mode TCD, lower ultrasound emission frequencies and multi-channel TCD. A 2-MHz digital Doppler with 33 sample gates placed with 2-mm spacing is configured to display Doppler signal power, colored red and blue for directionality, in an M-mode format. The spectrogram from a user-selected depth is displayed simultaneously [49]. Power M-mode TCD facilitates window location and alignment of the ultrasound beam to view blood flow from multiple vessels simultaneously, without sound or spectral clues. Lower ultrasound emission frequencies may lead to a higher signal intensity and thus sensitivity for detecting microemboli signal. Multi-channel TCD gives faint microemboli signal more opportunities to overcome the detection threshold.

\section{Conclusions}

TCD is highly suited to evaluate collateral circulation in carotid and intracranial stenoocclusive disease on account of its widespread availability and unique capacity to study realtime hemodynamics. It can reliably assess collateral flow via the circle of Willis and reversed OA flow in proximal ICA occlusion or severe stenosis, leptomeningeal collaterals in MCA occlusion, reversed BA flow in proximal BA occlusion and reversed VA flow in proximal subclavian or innominate artery occlusion. The intention of TCD is not to replace other angiographic imaging techniques, but to offer a wider diagnostic arsenal available for the physician in the acute management of suspected carotid and intracranial steno-occlusive disease. However, TCD screening can help to reduce the number of negative invasive angiograms. Advances in technology will focus on simplifying bone-window determination and limiting the operator dependency of the technique. 
Guan et al.: Usefulness of Transcranial Doppler Ultrasound in Evaluating

Cervical-Cranial Collateral Circulations

\section{References}

1 Liebeskind DS: Collateral circulation. Stroke 2003;34:2279-2284.

- 2 van Everdingen KJ, Visser GH, Klijn CJ, Kappelle LJ, van der Grond J: Role of collateral flow on cerebral hemodynamics in patients with unilateral internal carotid artery occlusion. Ann Neurol 1998;44:167-176.

- 3 Lau AY, Wong EH, Wong A, Mok VC, Leung TW, Wong KS: Significance of good collateral compensation in symptomatic intracranial atherosclerosis. Cerebrovasc Dis 2012;33:517-524.

- 4 Lima FO, Furie KL, Silva GS, Lev MH, Camargo EC, Singhal AB, Harris GJ, Halpern EF, Koroshetz WJ, Smith WS, Yoo AJ, Nogueira RG: The pattern of leptomeningeal collaterals on CT angiography is a strong predictor of long-term functional outcome in stroke patients with large vessel intracranial occlusion. Stroke 2010;41: 2316-2322.

5 Liebeskind DS, Cotsonis GA, Saver JL, Lynn MJ, Turan TN, Cloft HJ, Chimowitz MI, Warfarin-Aspirin Symptomatic Intracranial Disease (WASID) Investigators: Collaterals dramatically alter stroke risk in intracranial atherosclerosis. Ann Neurol 2011:69:963-974.

6 Awad I, Little JR, Modic MT, Furlan AJ: Intravenous digital subtraction angiography: an index of collateral cerebral blood flow in internal carotid artery occlusion. Stroke 1982;13:469-472.

- 7 Zbornikova V, Elfstrom J, Lassvik C, Johansson I, Olsson JE, Bjornlert U: Restenosis and occlusion after carotid surgery assessed by duplex scanning and digital subtraction angiography. Stroke 1986;17:1137-1142.

- 8 Report of American Academy of Neurology, Therapeutics and Technology Assessment Subcommittee: Assessment: transcranial Doppler. Neurology 1990;40:680-681.

- 9 Hendrikse J, Klijn CJ, van Huffelen AC, Kappelle LJ, van der Grond J: Diagnosing cerebral collateral flow patterns: accuracy of non-invasive testing. Cerebrovasc Dis 2008;25:430-437.

10 Markus HS, MacKinnon A: Asymptomatic embolization detected by Doppler ultrasound predicts stroke risk in symptomatic carotid artery stenosis. Stroke 2005;36:971-975.

11 Wilterdink JL, Feldmann E, Furie KL, Bragoni M, Benavides JG: Transcranial Doppler ultrasound battery reliably identifies severe internal carotid artery stenosis. Stroke 1997;28:133-136.

$\checkmark 12$ Zbornikova V: Long term follow-up of unilateral occlusion of the internal carotid artery including repeated tests of vasomotor reactivity by transcranial Doppler. Neurol Res 2006;28:220-224.

13 Kimiagar I, Bass A, Rabey JM, Bornstein NM, Gur AY: Long-term follow-up of patients with asymptomatic occlusion of the internal carotid artery with good and impaired cerebral vasomotor reactivity. Eur J Neurol 2010;17:1285-1290.

14 Alexandrov AV, Sloan MA, Wong LK, Douville C, Razumovsky AY, Koroshetz WJ, Kaps M, Tegeler CH: American Society of Neuroimaging Practice Guidelines Committee. Practice standards for transcranial Doppler ultrasound: part I - test performance. J Neuroimaging 2007;17:11-18.

15 Qureshi AI, Alexandrov AV, Tegeler CH, Hobson RW, Dennis Baker J, Hopkins LN: Guidelines for screening of extracranial carotid artery disease: a statement for healthcare professionals from the multidisciplinary practice guidelines committee of the American Society of Neuroimaging and cosponsored by the Society of Vascular and Interventional Neurology. J Neuroimaging 2007;17:19-47.

16 Jatuzis D, Zachrisson H, Blomstrand C, Ekholm S, Holm J, Volkmann R: Evaluation of posterior cerebral artery blood flow with transcranial Doppler sonography: value and risk of common carotid artery compression. J Clin Ultrasound 2000;28:452-460.

17 Müller M, Hermes M, Brückmann H, Schimrigk K: Transcranial Doppler ultrasound in the evaluation of collateral blood flow in patients with internal carotid artery occlusion: correlation with cerebral angiography. AJNR Am J Neuroradiol 1995;16:195-202.

-18 Zachrisson H, Fouladiun M, Blomstrand C, Holm J, Volkmann R: Functional assessment of high-grade ICA stenosis with duplex ultrasound and transcranial Doppler. Clin Physiol Funct Imaging 2012, 32:241-246.

19 Anzola GP, Gasparotti R, Magoni M, Prandini F: Transcranial Doppler sonography and magnetic resonance angiography in the assessment of collateral hemispheric flow in patients with carotid artery disease. Stroke 1995;26:214-217.

20 Miralles M, Dolz JL, Cotillas J, Aldoma J, Santiso MA, Giménez A, Capdevila A, Cairols MA: The role of the circle of Willis in carotid occlusion: assessment with phase contrast MR angiography and transcranial duplex. Eur J Vasc Endovasc Surg 1995;10:424-430.

-21 Christou I, Felberg RA, Demchuk AM, Grotta JC, Burgin WS, Malkoff M, Alexandrov AV: A broad diagnostic battery for bedside transcranial Doppler to detect flow changes with internal carotid artery stenosis or occlusion. J Neuroimaging 2001;11:236-242.

-22 Hedera P, Bujdakova J, Traubner P: Effect of collateral flow patterns on outcome of carotid occlusion. Eur Neuro 1995;35:212-216.

23 Ringelstein EB, Weiller C, Weckesser M, Weckesser S: Cerebral vasomotor reactivity is significantly reduced in low-flow as compared to thromboembolic infarctions: the key role of circle of Willis. J Neurol Sci 1994;121: 103-109.

24 Kluytmans M, van der Grond J, van Everdingen KJ, Klijn CJM, Kappelle LJ, Viergever MA: Cerebral hemodynamics in relation to pattern of collateral flow. Stroke 1999;30:1432-1439.

25 Saqqur M, Demchuk AM, Hill MD, Dean N, Schebel M, Kennedy J, Barber PA, Shuaib A: Bedside emergency transcranial Doppler diagnosis of severe carotid disease using orbital window examination. J Neuroimaging 2005;15:138-143. 
26 Reynolds PS, Greenberg JP, Lien LM, Meads DC, Myers LG, Tegeler CH: Ophthalmic artery flow direction on color flow duplex imaging is highly specific for severe carotid stenosis. J Neuroimaging 2002;12:5-8.

27 Krapf H, Widder B: The supratrochlear artery as an indicator of cerebral hemodynamics in carotid occlusion. Ultraschall Med 1998;19:114-119.

28 Telman G, Kouperberg E, Sprecher E, Hoffman A, Yarnitsky D: Assessment of ophthalmic artery collateral pathway in the hemispheric cerebral hemodynamics in patients with severe unilateral carotid stenosis. Neurol Res 2003;25:309-311.

29 Rutgers DR, Klijn CJ, Kappelle LJ, van Huffelen AC, van der Grond J: A longitudinal study of collateral flow patterns in the circle of Willis and the ophthalmic artery in patients with a symptomatic internal carotid artery occlusion. Stroke 2000;31:1913-1920.

-30 Schneider PA, Rossman ME, Bernstein EF, Ringelstein EB, Otis SM: Noninvasive assessment of cerebral collateral blood supply through the ophthalmic artery. Stroke 1991;22:21-36.

-31 Drakou AA, Koutsiaris AG, Tachmitzi SV, Roussas N, Tsironi E, Giannoukas AD: The importance of ophthalmic artery hemodynamics in patients with atheromatous carotid artery disease. Int Angiol 2011;30:547-554.

32 Mattle H, Grolimund P, Huber P, Sturzenegger M, Zurbrügg HR: Transcranial Doppler sonographic findings in middle cerebral artery disease. Arch Neurol 1988;45:289-295.

33 Demchuk AM, Christou I, Wein TH, Felberg RA, Malkoff M, Grotta JC, Alexandrov AV: Specific transcranial Doppler flow findings related to the presence and site of arterial occlusion. Stroke 2000;31:140-146.

-34 Brass LM, Duterte DL, Mohr JP: Anterior cerebral artery velocity changes in disease of the middle cerebral artery stem. Stroke 1989;20:1737-1740.

-35 Zanette EM, Fieschi C, Bozzao L, Roberti C, Toni D, Argentino C, Lenzi GL: Comparison of cerebral angiography and transcranial Doppler sonography in acute stroke. Stroke 1989;20:899-903.

-36 Kaps M, Damian M, Teschendorf U, Dorndorf W: Transcranial Doppler ultrasound findings in middle cerebral artery occlusion. Stroke 1990;21:532-537.

-37 Kim YS, Meyer JS, Garami Z, Molina CA, Pavlovic AM, Alexandrov AV: Flow diversion in transcranial Doppler ultrasound is associated with better improvement in patients with acute middle cerebral artery occlusion. Cerebrovasc Dis 2006;21:74-78.

-38 KimY, Sin DS, Park HY, Park MS, Cho KH: Relationship between flow diversion on transcranial Doppler sonography and leptomeningeal collateral circulation in patients with middle cerebral artery occlusive disorder. J Neuroimaging 2009;19:23-26.

39 Zareie H, Quain DA, Parsons M, Inder KJ, McElduff P, Miteff F, Spratt NJ, Levi C: The influence of anterior cerebral artery flow diversion measured by transcranial Doppler on acute infarct volume and clinical outcome in anterior circulation stroke. Int J Stroke 2013;8:228-234.

-40 Alexandrov AV,Demchuk AM, Wein TH, Grotta JC: Yield of transcranial Doppler in acute cerebral ischemia. Stroke 1999;30:1604-1609.

41 McVerry F, Liebeskind DS, Muir KW: Systematic review of methods for assessing leptomeningeal collateral flow. AJNR Am J Neuroradiol 2012;33:576-582.

-42 Brandt T, Knauth M, Wildermuth S, Winter R, von Kummer R, Sartor K, Hacke W: CT angiography and Doppler sonography for emergency assessment in acute basilar artery ischemia. Stroke 1999;30:606-612.

43 Ribo M, Garami Z, Uchino K, Song J, Molina CA, Alexandrov AV: Detection of reversed basilar flow with powermotion Doppler after acute occlusion predicts favorable outcome. Stroke 2004;35:79-82.

44 Sharma VK, Teoh HL, Chan BP, Ong BK: Reversed flow in the basilar artery in acute vertebrobasilar ischemia. J Clin Neurosci 2009;16:1493-1495.

-45 Klingelhöfer J, Conrad B, Benecke R, Frank B: Transcranial Doppler ultrasonography of carotid-basilar collateral circulation in subclavian steal. Stroke 1988;19:1036-1042.

46 Lee SI, Yang HD, Son IH, Han SJ: Recovery of reversed basilar artery flow as seen by transcranial sonography and MRA source images for vertebral dissection. J Neuroimaging 2008;18:451-453.

47 Huang YN, Gao S, Wang B, Li SW: The evaluation of intra- and extra-cranial circulation insubclavian steal syndrome. Chin Med J 1997;110:286-288.

48 Garami Z, Alexandrov AV: Neurosonology. Neurol Clin 2009;27:89-108.

49 Saqqur M, Hill MD, Alexandrov AV, Roy J, Schebel M, Krol A, Garami Z, Shuaib A, Demchuk AM: Derivation of power M-mode transcranial Doppler criteria for angiography proven MCA occlusion. J Neuroimaging 2006; $16: 323-328$ 